Case Report

\title{
Protracted Clonal Trajectory of a JAK2 V617F-Positive Myeloproliferative Neoplasm Developing during Long-Term Remission from Acute Myeloid Leukemia
}

\author{
Stephen E. Langabeer ${ }^{1},{ }^{1}$ Karl Haslam $\left(\mathbb{D},{ }^{1}\right.$ Maria Anne Smyth, ${ }^{2}$ John Quinn, \\ and Philip T. Murphy ${ }^{2}$ \\ ${ }^{1}$ Cancer Molecular Diagnostics, St. James's Hospital, Dublin 8, Ireland \\ ${ }^{2}$ Department of Haematology, Beaumont Hospital, Dublin 9, Ireland
}

Correspondence should be addressed to Stephen E. Langabeer; slangabeer@stjames.ie

Received 27 February 2018; Accepted 31 March 2018; Published 9 May 2018

Academic Editor: Sudhir Tauro

Copyright (C) 2018 Stephen E. Langabeer et al. This is an open access article distributed under the Creative Commons Attribution License, which permits unrestricted use, distribution, and reproduction in any medium, provided the original work is properly cited.

\begin{abstract}
Although transformation of the myeloproliferative neoplasms (MPNs) to acute myeloid leukemia (AML) is well documented, development of an MPN in patients previously treated for, and in remission from, AML is exceedingly rare. A case is described in which a patient was successfully treated for AML and in whom a JAK2 V617F-positive MPN was diagnosed after seven years in remission. Retrospective evaluation of the JAK2 V617F detected a low allele burden at AML diagnosis and following one course of induction chemotherapy. This putative chemoresistant clone subsequently expanded over the intervening seven years, resulting in a hematologically overt MPN. As AML relapse has not occurred, the MPN may have arose in a separate initiating cell from that of the AML. Alternatively, both malignancies possibly evolved from a common precursor defined by a predisposition mutation with divergent evolution into MPN through acquisition of the JAK2 V617F and AML through acquisition of different mutations. This case emphasizes the protracted time frame from acquisition of a disease-driving mutation to overt MPN and further underscores the clonal complexity in MPN evolution.
\end{abstract}

\section{Introduction}

The clonal evolution of a myeloproliferative neoplasm (MPN) into acute myeloid leukemia (AML) is complex: both the JAK2 V617F and CALR exon nine mutations are absent in a significant number of MPN patients evolving to AML implicating the transformation of a common myeloid progenitor at a stage before acquisition of the MPN driver mutation or evolution from a distinct initiating precursor from that harboring the JAK2 V617F or CALR mutation [1-3]. Furthermore, not only the type of additional mutations but also the order in which these mutations are acquired can influence clonal evolution and phenotype [4]. In contrast, the emergence of a JAK2 V617F-positive MPN on the background of AML is exceedingly rare. Nearly all those cases reported have occurred in AML patients in long-term remission after chemotherapy or autologous stem cell transplantation and where retrospective genotyping of the AML presentation material was performed, demonstrating the absence of the JAK2 V617F mutation [5-8]. Molecular investigation of another case suggested that the MPN may be secondary to the AML [9]. A case is described of a patient developing a JAK2 V617F-positive MPN when in remission of AML for seven years and in whom retrospective molecular investigation was able to unravel the evolutionary trajectory of the MPN.

\section{Case Report}

A 64-year-old female presented in April 2010 with a twoweek history of lethargy. A full blood count revealed a hemoglobin count of $8.6 \mathrm{~g} / \mathrm{dL}$, a platelet count of $48 \times 10^{9} / \mathrm{L}$, and a white cell count of $96.4 \times 10^{9} / \mathrm{L}$ with a large population of blasts. On physical examination, there was no 


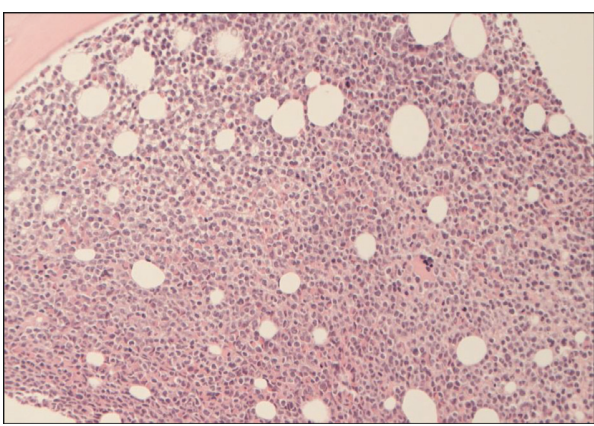

(a)

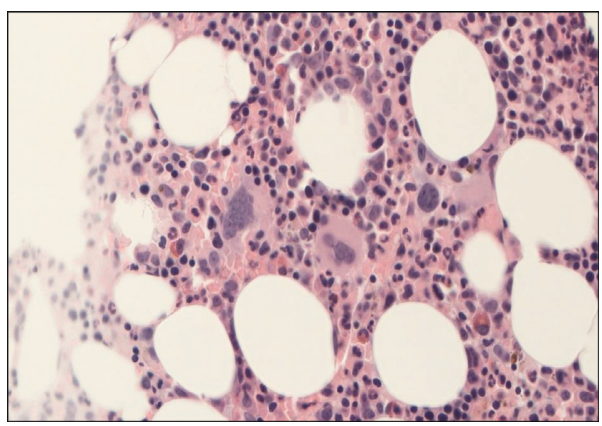

(c)

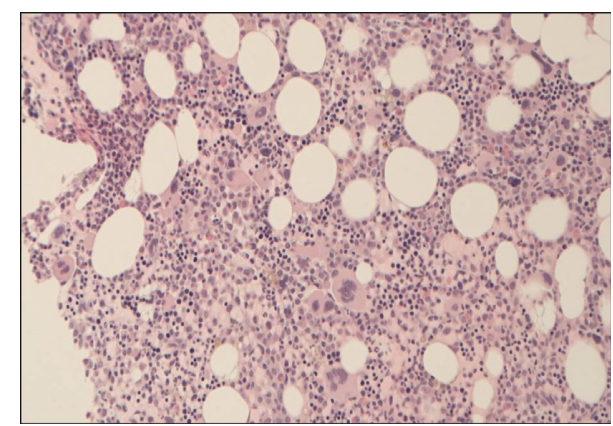

(b)

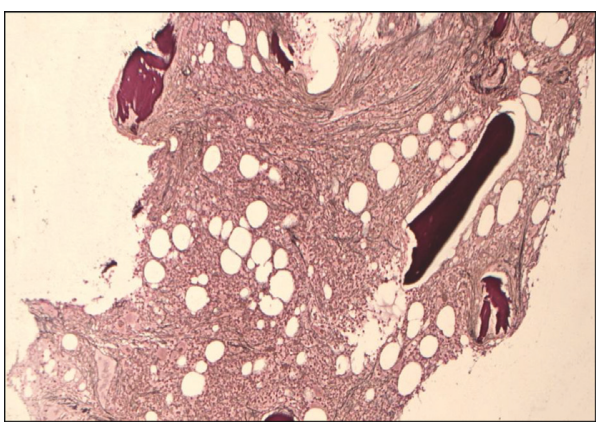

(d)

Figure 1: Bone marrow biopsy (a) at diagnosis of acute myeloid leukemia (AML) demonstrating infiltration by myeloblasts; (b) after one course of AML therapy showing atypical megakaryocyte morphology and focal clustering; (c) at diagnosis of myeloproliferative neoplasm (MPN) with myeloid hypercellularity and increased megakaryocytes; and (d) at diagnosis of MPN demonstrating increased reticulin deposition.

palpable liver or spleen. Peripheral blood immunophenotyping showed two blast populations: one population $(20 \%)$ positive for HLA-DR, CD117, CD33, and CD13 and a second population (48\%) positive for HLA-DR, CD33, CD13, CD14, and CD15. The bone marrow aspirate was hypercellular with the vast majority of cells either myeloblasts or monoblasts, all consistent with a diagnosis of acute myeloid leukemia (AML) of myelomonocytic type (Figure 1(a)). Cytogenetic analysis demonstrated a normal karyotype, and there was no evidence of an FLT3 internal tandem duplication mutation. The patient achieved a morphological remission after one course of daunorubicin and cytarabine $(3+10)$. The bone marrow trephine biopsy at this time showed no evidence of blasts but remained hypercellular with some abnormal megakaryocyte forms showing focal clustering (Figure 1(b)). The reticulin stain was normal. The patient completed three courses of consolidation chemotherapy ending in August 2010 with hemoglobin, hematocrit, red cell count, and platelets coming into normal range within two years of finishing AML treatment and remains in remission.

A mild neutrophilia $\left(7.85 \times 10^{9} / \mathrm{L}\right)$ was noted in October 2013 and an eosinophilia $\left(0.4 \times 10^{9} / \mathrm{L}\right)$ was noted in January 2017 , slowly rising to $18.9 \times 10^{9} / \mathrm{L}$ neutrophils and $0.6 \times 10^{9} / \mathrm{L}$ eosinophils. Qualitative molecular analysis of peripheral blood in July 2017 showed no evidence of BCR-ABL1 transcripts but detected the presence of the JAK2 V617F mutation. Subsequent bone marrow aspirate and biopsy in August 2017 (seven years after completion of AML therapy) revealed hypercellularity with increased myelopoiesis undergoing normal maturation. Megakaryocytes were increased in number with some immature forms being seen and an increase in mature myeloid cells to erythroid cells (Figure 1(c)). Staining for reticulin demonstrated increased deposition (Figure 1(d)), resulting in a diagnosis of MPN unclassified. Currently, the patient is asymptomatic and is on no active therapy to control her white cell count.

Quantitative assessment of the JAK2 V617F allele burden was performed on the archival material using an assay previously described with a sensitivity of $0.01 \%$ mutant alleles [10]. Quantitative PCR demonstrated bone marrow allele burdens of $0.2 \%$ at AML diagnosis (in independent duplicate samples), 5.7\% after one course of AML therapy, and $72.7 \%$ at MPN diagnosis.

\section{Discussion}

Molecular analysis of AML derived from a JAK2 V617Fpositive MPN has demonstrated divergent pathways to transformation, highlighting the underlying clonal complexity of MPN evolution. In the case described herein, retrospective molecular investigation detected a low JAK2 V617F allele burden at AML diagnosis that was further unmasked by the AML induction chemotherapy. As AML relapse has not occurred, the MPN may have arose in a separate initiating cell from that of the AML. Alternatively, both malignancies possibly evolved from a common precursor defined by a predisposition mutation with divergent evolution into MPN or AML through acquisition of 


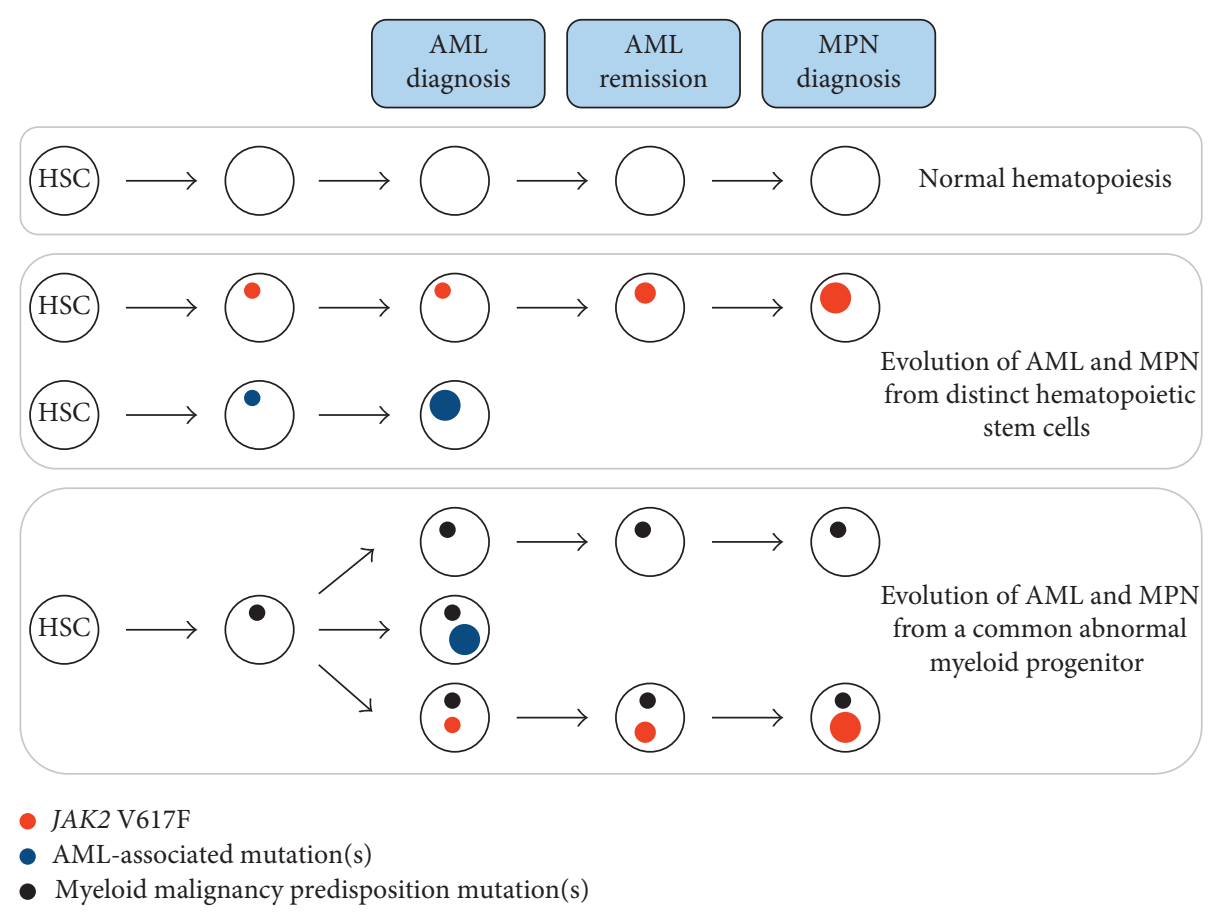

FIGURE 2: Possible divergent clonal trajectories of the JAK2 V617F-positive myeloproliferative neoplasm and acute myeloid leukemia in tandem with normal hematopoiesis. AML: acute myeloid leukemia; MPN: myeloproliferative neoplasm; HSC: hematopoietic stem cell.

the JAK2 V617F or AML-associated mutations, respectively (Figure 2). The chemoresistant JAK2 V617F clone [11] remained clinically silent for seven years, although continually expanding, until hematological and morphological diagnosis of an MPN with a high JAK2 V617F allele burden. The high JAK2 V617F allele burden likely represents loss of heterozygosity in at least some mutant clones, analogous to that observed in the progression of polycythemia vera [12]. This phenomenon of a minor JAK2 V617F clone at AML diagnosis has been described previously in a patient who developed polycythemia vera after five years in remission for AML treatment and in whom retrospective analysis revealed a JAK2 V617F allele burden of $2.0 \%$ at the time of AML diagnosis [13]. Whole-exome sequencing and cluster analysis in a recently reported similar case suggest that preleukemic hematopoietic stem cells of AML can not only contribute to myeloid hematopoiesis during remission but may also give rise to another malignancy such as MPN [14]. Moreover, although de novo JAK2 V617F-positive AML is uncommon, it can be associated with the $t(8 ; 21)$ and trisomy 8 cytogenetic abnormalities, with myelodysplastic changes, and a high allele burden $[15,16]$, features not observed in this case.

A protracted time course is clearly evident from acquisition of the initial JAK2 V617F driver mutation to development of a hematologically and clinically overt MPN accentuating the different kinetics of development of MPN and AML and suggesting that initiation of the MPN may have been an earlier event relative to that of the AML. Studies have shown that this process may take several years and have attempted to quantify the annual increase in JAK2 V617F allele burden [17]. A more recent study of long-term follow-up of blood donors who developed an MPN has shown a similar latency though individual variation does exist, which is possibly a reflection of both inherited and acquired genetic factors [18].

Finally, given the expanding focus in minimal residual disease-directed therapy for AML patients [19], caution is warranted in the interpretation of low-level mutations such as the JAK2 V617F, as these may not represent residual disease but a latent, coexisting hematological malignancy.

\section{Disclosure}

Current address of Karl Haslam is Genomics Medicine Ireland, Cherrywood Business Park, Dublin 18, Ireland.

\section{Conflicts of Interest}

The authors declare that there are no conflicts of interest regarding the publication of this paper.

\section{References}

[1] P. J. Campbell, E. J. Baxter, P. A. Beer et al., "Mutation of JAK2 in the myeloproliferative disorders: timing, clonality studies, cytogenetic associations, and role in leukemic transformation," Blood, vol. 108, no. 10, pp. 3548-3555, 2006.

[2] A. Theocharides, M. Boissinot, F. Girodon et al., "Leukemic blasts in transformed JAK2 V617F-positive myeloproliferative disorders are frequently negative for the JAK2 V617F mutation," Blood, vol. 110, no. 1, pp. 375-379, 2007.

[3] S. E. Langabeer, K. Haslam, and E. Elhassadi, "The mutant CALR allele burden in essential thrombocythemia at transformation to acute myeloid leukemia," Blood Cells, Molecules and Diseases, vol. 65, pp. 66-67, 2017. 
[4] C. A. Ortmann, D. G. Kent, J. Nangalia et al., "Effect of mutation order on myeloproliferative neoplasms," New England Journal of Medicine, vol. 372, no. 7, pp. 601-612, 2015.

[5] C. Chabannon, M. Bost, and D. Hollard, "A case of polycythemia vera occurring in a patient with acute nonlymphoblastic leukemia (ANLL) in long-term first complete remission," Leukemia, vol. 8, no. 7, pp. 1243-1244, 1994.

[6] A. R. Walker, P. G. Rothberg, and J. L. Liesveld, "A case of JAK2 positive essential thrombocythemia 16.5 years after autologous marrow transplantation for AML," Bone Marrow Transplantation, vol. 39, no. 11, pp. 725-726, 2007.

[7] E. Antonioli, P. Guglielmelli, G. Poli, V. Santini, A. Bosi, and A. M. Vannucchi, "Polycythemia vera following autologous transplantation for AML: insights on the kinetics of JAK2 V617F clonal dominance," Blood, vol. 110, no. 13, pp. 4620-4621, 2007.

[8] A. Belotti, E. Doni, E. Elli, V. Rossi, P. Pioltelli, and E. M. Pogliani, "Development of polycythemia vera after chemotherapy-induced remission of acute myeloid leukemia: a case report," Acta Haematologica, vol. 126, no. 1, pp. 52-53, 2011.

[9] S. Girsberger, A. Karow, P. Lundberg et al., "JAK2 V617Fmutated myeloproliferative neoplasia developing five years after wild-type JAK2 acute myeloid leukemia: a case report," Acta Haematologica, vol. 129, no. 1, pp. 23-25, 2013.

[10] K. Haslam, K. M. Molloy, E. Conneally, and S. E. Langabeer, "Evaluation of a JAK2 V617F quantitative PCR to monitor residual disease post-allogeneic hematopoietic stem cell transplantation for myeloproliferative neoplasms," Clinical Chemistry and Laboratory Medicine, vol. 52, no. 3, pp. e29-e31, 2014.

[11] E. Verger, E. Rattarittamrong, G. Letort, E. Raffoux, B. Cassinat, and J. J. Kiladjian, "Chemotherapy for post-myelofibrosis acute myeloid leukemia: eradication of the leukemic clone but not the MPN clone," Leukemia and Lymphoma, vol. 58, no. 3, pp. 749-751, 2017.

[12] A. M. Vannucchi, E. Antonioli, P. Guglielmelli, A. Pardanani, and A. Tefferi, "Clinical correlates of JAK2 V617F presence or allele burden in myeloproliferative neoplasms: a critical reappraisal," Leukemia, vol. 22, no. 7, pp. 1299-1307, 2008.

[13] C. A. Portell, M. A. Sekeres, H. J. Rogers, and R. V. Tiu, "De novo polycythemia vera arising 5 years following acute myeloid leukemia remission: suggestion of a chemotherapy resistant JAK2 clone," British Journal of Haematology, vol. 157, no. 2, pp. 266-267, 2012.

[14] S. Sato, H. Itonaga, M. Taguchi et al., "Clonal dynamics in a case of acute monoblastic leukemia that later developed myeloproliferative neoplasm," International Journal of Hematology, 2018, In press.

[15] S. Schnittger, U. Bacher, W. Kern, T. Haferlach, and C. Haferlach, "JAK2 V617F as a progression marker in CMPD and as a cooperative mutation in AML with trisomy 8 and $t(8$; 21): a comparative study on 1103 CMPD and 269 AML cases," Leukemia, vol. 21, no. 8, pp. 1843-1845, 2007.

[16] J. E. Hidlago-López, R. Kanagal-Shamanna, L. J. Medeiros et al., "Morphologic and molecular characteristics of de novo AML with JAK2 V617F mutation," Journal of the National Comprehensive Cancer Network, vol. 15, no. 6, pp. 790-796, 2017.

[17] C. Nielsen, S. E. Bojesen, B. G. Nordestgaard, K. F. Kofoed, and H. S. Birgens, "JAK2 V617F somatic mutation in the general population: myeloproliferative neoplasm development and progression rate," Haematologica, vol. 99, no. 9, pp. 1448-1455, 2014.

[18] T. McKerrell, N. Park, J. Chi et al., "JAK2 V617F hematopoietic clones are present several years prior to MPN diagnosis and follow different expansion kinetics," Blood Advances, vol. 1, no. 14, pp. 968-971, 2017.
[19] C. S. Hourigan, R. P. Gale, N. J. Gormley, G. J. Ossenkoppele, and R. B. Walter, "Measurable residual disease testing in acute myeloid leukemia," Leukemia, vol. 31, no. 7, pp. 1482-1490, 2017. 


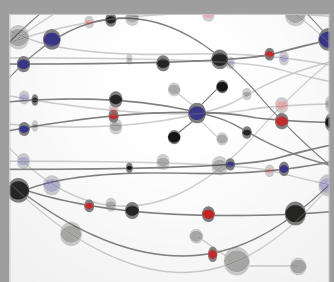

The Scientific World Journal
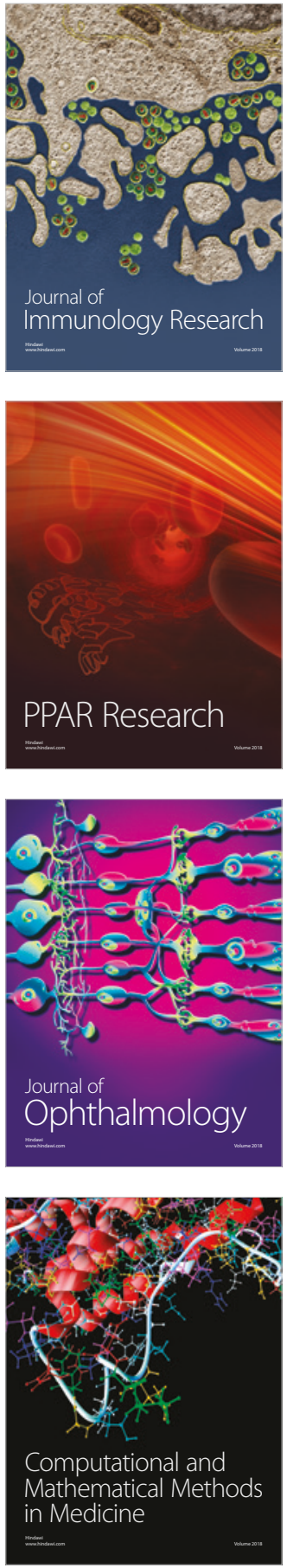

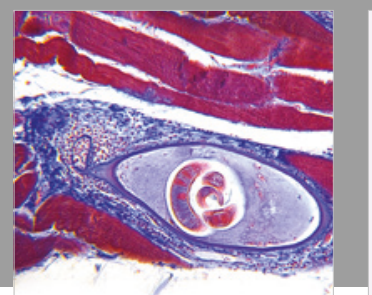

Gastroenterology Research and Practice

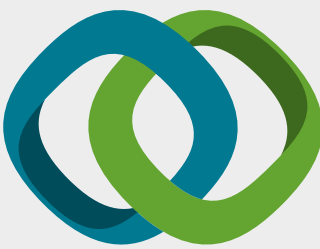

\section{Hindawi}

Submit your manuscripts at

www.hindawi.com
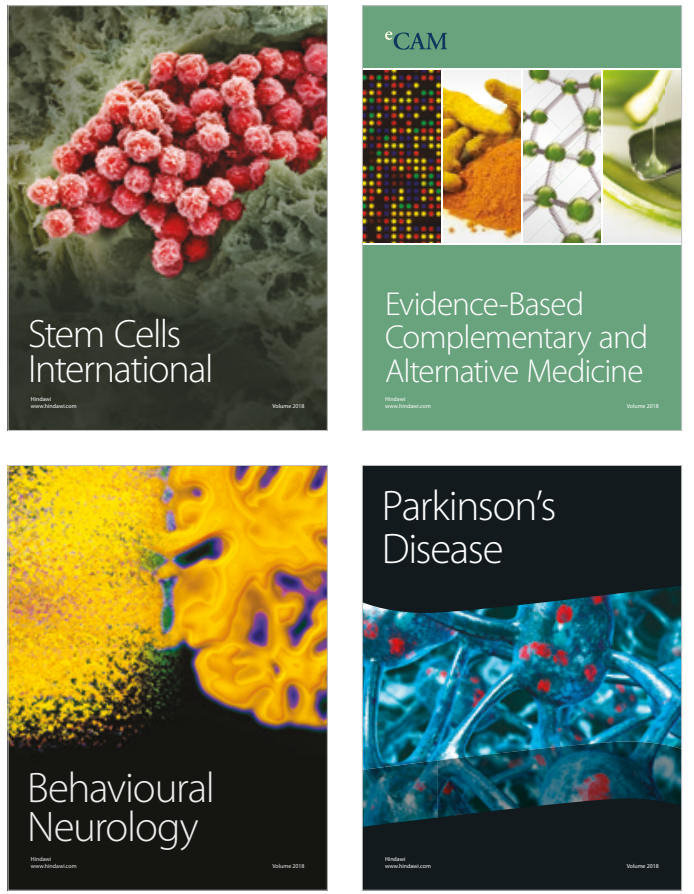

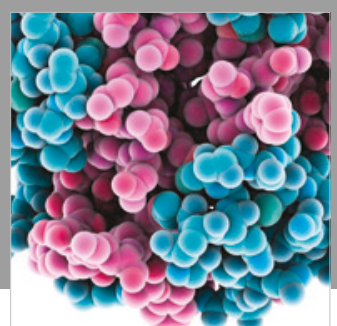

ournal of

Diabetes Research

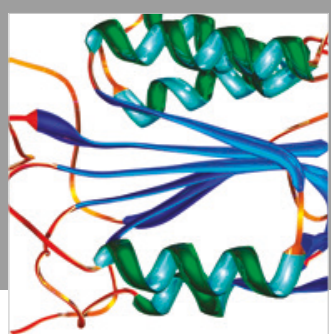

Disease Markers
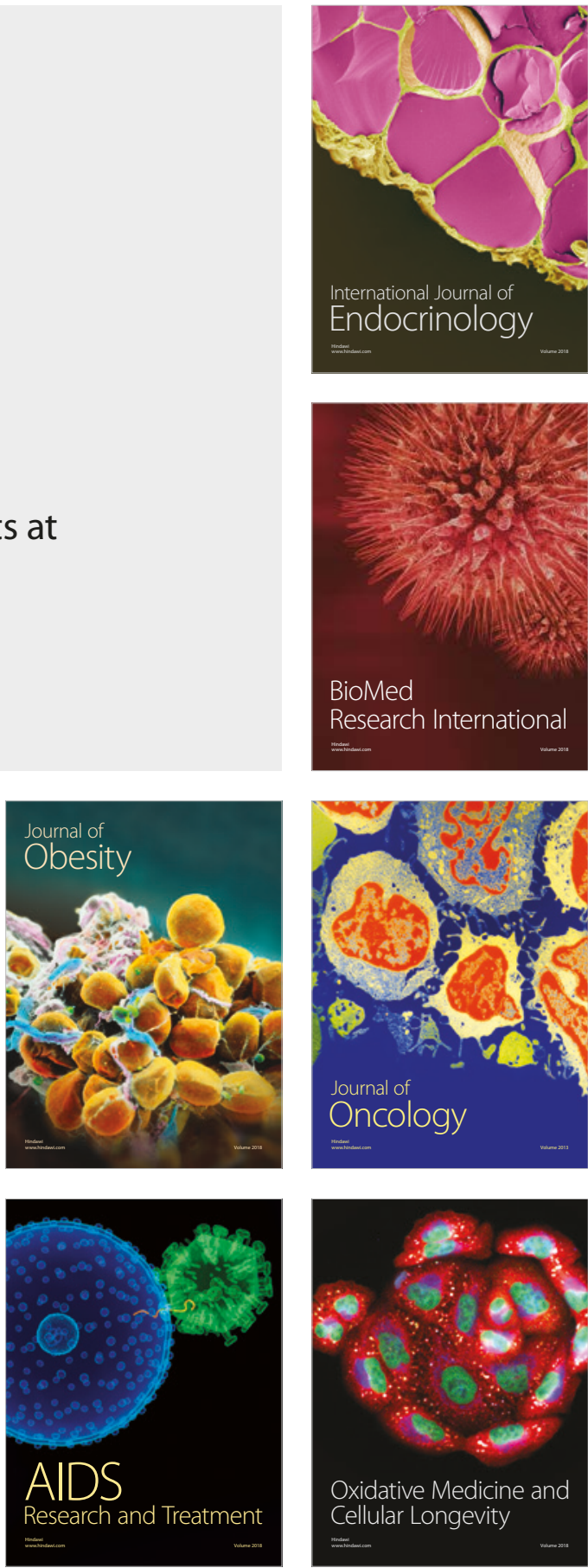